\title{
Effect of Haemophilus influenzae Type b Vaccination on Nasopharyngeal Carriage Rate in Children, Tehran, 2019
}

\author{
Sedigheh Rafiei Tabatabaei, ${ }^{1}$ Sara Mohammadzadeh, ${ }^{1}$ Seyed Mohsen Zahraei, ${ }^{2}$ \\ Sussan Mahmoudi, ${ }^{2}$ Ghazaleh Ghandchi, ${ }^{1}$ Seyedeh Mahsan Hoseini-Alfatemi ${ }^{D},{ }^{1}$ \\ Abdollah Karimi, ${ }^{1}$ and Ahmadreza Shamshiri ${ }^{3}$ \\ ${ }^{1}$ Pediatric Infections Research Center, Research Institute for Children's Health, Shahid Beheshti University of Medical Sciences, \\ Tehran, Iran \\ ${ }^{2}$ Center for Communicable Disease Control, Ministry of Health and Medical Education, Tehran, Iran \\ ${ }^{3}$ Department of Epidemiology and Biostatistics, School of Public Health, Tehran University of Medical Sciences, Tehran, Iran
}

Correspondence should be addressed to Seyedeh Mahsan Hoseini-Alfatemi; mahsan.hoseinialfatemi@gmail.com

Received 29 June 2020; Revised 21 February 2021; Accepted 8 March 2021; Published 16 March 2021

Academic Editor: Cecilia Trucchi

Copyright (c) 2021 Sedigheh Rafiei Tabatabaei et al. This is an open access article distributed under the Creative Commons Attribution License, which permits unrestricted use, distribution, and reproduction in any medium, provided the original work is properly cited.

Background. Haemophilus influenzae (H. influenzae) strains, which commonly reside as commensals within the human pharynx and can remain as an asymptomatic carrier, but become invasive leading to pneumonia, septic arthritis, or meningitis. The Pentavac (pentavalent vaccine, manufactured by India, SII (DTwP-HepB-Hib)) was introduced to the Iranian National Immunization Plan in November 2014. The aim of this study is to investigate $H$. influenzae type b (Hib) carrier rate among children under 6 years old in Tehran. Methods. This cross-sectional study was performed on 902 children including vaccinated/unvaccinated in the age of 6 months to 6 years, in Tehran. Sampling was performed from July 2019 to September 2019. Nasopharyngeal samples were taken from children by sterile swab. The PCR method was used to extract DNA. Then, all $H$. influenzae isolates were initially confirmed by molecular tests. BexA was used to distinguish typeable $H$. influenzae strains from nontypeable Haemophilus influenzae (NTHi). Results. A total of 902 children were enrolled in the study: 452 were female (51\%). H. influenzae carriage rate was 267 (29\%), of that 150 samples (16.6\%) were typeable. The nasopharyngeal Hib carrier rate in the children was 2.6\% (24/902). 262 cases did not receive Hib vaccine. Analysis in nonnursery's children aged 4 to 6 (unvaccinated) years showed that the lower educational level of father, mother, and family number correlated with increased odds of colonization of children with Hib. Conclusion. Our findings showed a significant decrease (60\%) in the overall Hib nasopharyngeal carriage in healthy children under six years after 5 years after the start of Hib vaccination.

\section{Introduction}

H. influenzae is a Gram-negative Coccobacillus that is commonly found as part of the natural flora of the upper respiratory tract in humans, especially in healthy children [1]. These bacteria can be colonized in the throat of healthy children as an asymptomatic carrier and persist for several months, and intravascular invasion colonization of this bacterium can cause severe invasive diseases such as meningitis, septic arthritis, epiglottitis, purulent pericarditis, and pneumonia, especially in children under 5 years [1-3].
According to the type of capsular polysaccharides, these bacteria are classified to six serotypes called a, b, c, d, e, and $\mathrm{f}$, identified by agglutination in the presence of a specific serum for each serotype [4], and one type without capsule or nontypeable $\mathrm{H}$. influenzae (NTHi) that cannot be serotyped by formal agglutination with a specific antiserum [5].

The nasopharyngeal carries of the organism play an important role in the transmission of $\mathrm{Hib}$, the pathogenesis of Hib diseases, and the emergence of immunity against bacteria. Then, this is colonized for a long time and caused 
respiratory tract infection, or entered the bloodstream when passing through the mucosa, and caused invasive diseases [1, 2].

According to the World Health Organization (WHO) report, in 2000, one of the two main causes of preventable diseases in children under 5 was $H i b$ [2]. Carrier rate of $H$. influenzae in vaccinated children of Nepal reported 5\% [6].

$\mathrm{Hib}$ vaccination coverage increased in recent years. Since 2013 , about $98 \%$ of the WHO member states and $52 \%$ of infants, globally, received $H i b$ vaccine by immunization programs [7].

The Hib Vaccination decreases the colonization of the Hib, and several studies have shown that other serotypes increase [2]. Currently, protein-polysaccharide conjugate $\mathrm{Hib}$ vaccines have been incorporated into the routine immunization program in almost all countries around the world [8]. Hib carrier rate in healthy children under five years in Brazil was rare after 10 years of the Hib vaccine [9].

Studies in different countries have shown that introduction of the $\mathrm{Hib}$ vaccination has reduced $\mathrm{Hib}$ carrier, in both developed and developing countries [3, 8, 10-16]. The Pentavac (pentavalent vaccine, manufactured by India, SII) entered the Iranian National Immunization Plan, in November 2014, that includes diphtheria, tetanus, whole cell pertussis, hepatitis $\mathrm{B}$, and $H$. influenza type $b$ vaccines administered three times at months 2, 4, and 6 of infants' age [17].

Therefore, the evaluation of nasopharyngeal colonization of vaccinated children can help us to determine the extent of the effect of $H i b$ vaccine on this colonization compared to the similar study before vaccination. In this study, we aimed to investigate the prevalence of Hib carrier in healthy children under 6 years of age.

\section{Subjects, Materials, and Methods}

The present cross-sectional study was conducted in four health centers of northern Tehran (Ershad, Sheibani, Torab, and Samarghandi). Sampling was performed on vaccinated $>$ (Pentavac vaccine, India, SII (DTwP-HepB-Hib)) and unvaccinated children 6 months to 6 years of age. Sampling was carried out continuously, in health centers in northern Tehran from July to September 2019. Children's information is collected by using a questionnaire. Parental oral consent was obtained. None of the participants included in our study had any type of illness on the date of specimen collection and none of them had received antibiotic therapy in preceding 4 weeks. Children who were ill at the time of the study were excluded. Nasopharyngeal samples were collected with sterile cotton swabs and immediately dropped in TSB medium then referred to the laboratory of the Pediatric Infections Research Center of the Mofid Children's Hospital.

The study protocol was approved by Pediatric Infections Research Center (PIRC) of Shahid Beheshti University of Medical Sciences. The ethical approval code is No. IR.SBMU.MSP.REC.1395.551.

2.1. PCR and Molecular Identification. The molecular identification of $H$. Influenzae samples was confirmed by using PCR and amplification of the omp6 gene by specific gene primers. First, the DNA extraction method of bacterial nucleic acids was based on the DNA Kit protocol (Sinaclon, Iran) and DNA extraction product was stored in a $-70^{\circ} \mathrm{C}$ freezer. H. Influenzae ATCC 49766 was used as positive control for Hib. Finally, PCR was used for serotyping and determination of $\mathrm{Hib}$. PCR procedure was performed as follows: an initial denaturation at $95^{\circ} \mathrm{C}$ for $5 \mathrm{~min}, 40$ cycles of denaturation at $95^{\circ} \mathrm{C}$ for $30 \mathrm{~s}$, annealing at $58^{\circ} \mathrm{C}$ for $30 \mathrm{~s}$, elongation at $72^{\circ} \mathrm{C}$ for $45 \mathrm{~s}$, and a final extension step at $72^{\circ} \mathrm{C}$ for $7 \mathrm{~min}$. The resulting PCR products were stained with Red safe (cat no: 1005.50) for $20 \mathrm{~min}$. Then, specific bands were visualized on gel electrophoresis of PCR products under UV illumination.

To confirm species identification, PCR amplification was performed to detect the omp6 gene from genomic DNA [18]. Additionally, the PCR amplification was further carried out using the primer pair specific to bexA (a fragment of $354 \mathrm{bp}$ in length) genes to distinguish encapsulated $H$. influenzae strains from NTHi strains. To determine the capsular genotypes, or phylogenetic relationships, of the Hib strains, PCR amplification was performed with two sets of primers designed by Ueyama et al. [18].

2.2. Statistical Analysis. Categorical data were reported as raw and relative abundance and quantitative data assuming normal distribution as mean and standard deviation. Simple and multiple logistic regression was used to analyze factors associated with colonization frequency with Hib (two-state qualitative variable). Given that the day-nursery variable was identified as an effect modifier, data analysis was performed separately for nursery's children and nonnursery's children. Statistical significance was considered less than 0.05.

\section{Results}

3.1. Descriptive Data. The demographic and baseline values of samples are presented in Table 1. A total of 902 children were enrolled in the study: 452 were female (51\%) and 450 were male.

146 sample of children under 1 year, 154 samples under 1-2 years, 153 samples under 2-3 years, 150 samples under 3-4 years, 151 samples under 4-5 years, and 148 samples under 5-6 years were taken. Given that the vaccine program has been started in 2014 in Iran, children over the age of 4 and some children in other age groups did not receive the vaccine. So, because of the starting time of the vaccination and some other reasons, 262 cases have not received Hib vaccine, which includes 6 children (one year old), 5 children (two years old), 16 children (three years old), 34 children (four years old), 56 children (five years old), and 145 children (six years old).

In PCR assays, 267 (29\%) of the 902 samples were $H$. influenzae, of that 150 (56.17\%) encapsulated and type-able isolates have been reported. Among the 640 children who received the vaccine and among the 262 who did not receive vaccine, 15 $(2.3 \%)$ and $9(3.4 \%)$, respectively, were reported as Hib. 3.2. Factors Associated with Colonization of Haemophilus
influenzae Type $b$. The mean age in colonized children was 
TABLE 1: The demographic and baseline values of samples.

\begin{tabular}{|c|c|c|}
\hline Variables & & $N(\%)$ \\
\hline Age* $^{*}$ & & $3.50 \pm 1.70$ \\
\hline \multirow{2}{*}{ Gender } & Female & $452(50.11 \%)$ \\
\hline & Male & $450(49.89 \%)$ \\
\hline \multirow{3}{*}{ Family number } & $2-3$ & $481(53.33)$ \\
\hline & 4 & $356(39.47)$ \\
\hline & $\geq 5$ & $65(7.21)$ \\
\hline \multirow[t]{2}{*}{ Father education } & $\begin{array}{l}\text { Associate's degree, } \\
\text { diploma, or less }\end{array}$ & $296(32.82 \%)$ \\
\hline & BSc, MSc, or doctorate & $606(67.18 \%)$ \\
\hline \multirow[t]{2}{*}{ Mother education } & $\begin{array}{l}\text { Associate's degree, } \\
\text { diploma, or less }\end{array}$ & $284(31.49 \%)$ \\
\hline & BSc, MSc or doctorate & $618(68.51 \%)$ \\
\hline \multirow{2}{*}{$\begin{array}{l}\text { Pentavalent vaccine } \\
\text { history }\end{array}$} & No & $262(29.05 \%)$ \\
\hline & Yes & $640(70.95 \%)$ \\
\hline \multirow{2}{*}{$\begin{array}{l}\text { Babysitting at home or } \\
\text { day-nursery }\end{array}$} & Home & $539(59.76 \%)$ \\
\hline & Day-nursery & $363(40.24 \%)$ \\
\hline \multirow{2}{*}{$\begin{array}{l}\text { Smoking (adults, past or } \\
\text { current) }\end{array}$} & No & $795(88.14 \%)$ \\
\hline & Yes & $107(11.86 \%)$ \\
\hline
\end{tabular}

${ }^{*}$ Mean $\pm \mathrm{SD}$.

$4.25 \pm 1.29$ and in non-colonized children was $3.48 \pm 1.70$. As shown in the table, in the data analysis (multivariable), the only variable that has a significant association with colonization of $\mathrm{Hib}$ is the level of maternal education.

3.3. Data Analysis in Nursery's Children. Data on nursery's children are presented in Table 2. As shown in this table, none of the variables studied in preschool children had a significant relationship with the colonization of $\mathrm{Hib}$.

3.4. Analysis in Nonnursery's Children. Multivariable analysis showed that aged 4 to 6 years (compared to age 1 to 3 years) (adjusted $\mathrm{OR}=4.25 ; 95 \% \mathrm{CI}: 1.11-16.33 ; p$ value $=0.04$ ), lower educational level of father (adjusted $\mathrm{OR}=3.72 ; 95 \%$ CI: $0.97-14.32 ; p$ value $=0.056$ ), and family number (adjusted $\mathrm{OR}=3.72 ; 95 \% \mathrm{CI}: 0.97-14.32 ; p$ value $=0.056)$ correlated with increased odds of colonization of nonnursery's children with Hib. This analysis shows that statistically, a mother's level of education is as effective as a father's level of education (adjusted OR $=3.64 ; 95 \%$ CI: $0.94-14.02 ; p$ value $=0.061$ ), approximately. The analysis of data in nonnursery's children is summarized in Table 3.

The day-nursery variable is an effect modifier in this study. Therefore, the data of nursery's children and nonnursery's children were analyzed separately. None of the variables studied in nursery's children had a significant relationship with the colonization of Hib.

\section{Discussion}

This study was conducted to investigate the prevalence of colonization of $H$. influenzae after 5-year vaccination in children under six years old, in Tehran.
The findings of this study showed that nonnursery's children aged 4 to 6 years, with low mother and father education level, and family members more than 5 are more at risk for colonization of $H$. influenzae. Carrier rate of $\mathrm{Hib}$ varies greatly from place to place.

In this study, the nasopharyngeal $\mathrm{Hib}$ carriage rate in the children was $2.6 \%$. This is similar to reports from other developing countries in CDC reports $(0.5 \%-3 \%)$ [7]. This prevalence is higher than the Hib colonization rate in some developed countries such as Canada [19], UK [13], Japan [20], and Brazil [21] after vaccination (0\%-1.5\%) [21]. In these countries following the introduction of routine immunization of infants with $\mathrm{Hib}$ conjugate vaccines, the carrier rate has reduced dramatically, in both vaccinated and unvaccinated children due to the herd effect [22]. On the contrary, in nasopharyngeal carriage studies using culture and identification of all $H$. influenzae serotypes before vaccination, carriage rates vary from $15 \%$ to $40 \%[23,24]$. It seems these findings are more than this study based on our data that no clear explanation has been provided. One study suggested that oropharyngeal culture for Hib carriers is more sensitive than nasopharyngeal culture. Samples were obtained from 717 healthy children younger than 6 by a single oropharyngeal swab from each participant and to streak into chocolate blood agar. The PCR was used to determine capsular genotype. The overall rate of $H$. influenzae carriage in vaccinated and unvaccinated children was $14.1 \%$. Age, the place of study, presence of young-siblings, and complete Hib vaccination status were associated with colonization, independently [22]. In Karimi et al.'s study in Tehran in 2007 before vaccination, out of 1000 children aged $\leq 5$ years from 25 daycare centers, the rate of Hib carriage by a culture method was reported as $7.6 \%$ [25]. This study approved that the vaccination has been able to reduce about $60 \%$ of the pharyngeal carrier. Karimi et al.'s result was an important estimation for determining the status of Hib colonization in Iranian children before Hib vaccination [2].

One study in Kenya showed immunization using Hib conjugate vaccines declined the prevalence of nasopharyngeal carriage [10]. In another study, immunization with $\mathrm{Hib}$ conjugate vaccines has also decreased the rate of colonization to less than 1\% [14]. In Giufre et al.'s study in Italy in 2015, almost all $\mathrm{H}$. influenza isolates in healthy children were $\mathrm{NTHi}$ type except 3 capsuled isolates that did not belong to vaccinepreventable serotypes. According to the results of the study, vaccination reduced or eliminated the $\mathrm{Hib}$ carriage [22]. In the current study, all children were selected from a climatic zone north of Tehran, so further investigation is needed to obtain more accurate results. In addition, in previous studies, the age and presence of young siblings were identified as independent risk factors [22]. In a study by Odutola et al. in 2013, on 1030 Gambian infants (median age 35 weeks), the little effect of age on carriage of any of the potential pathogens was observed, and with the increase of age, the prevalence of carriage with $H$. influenzae trends to increase. The finding of this study is similar to Odutola et al.'s study. Based on these findings, the mean age in colonized children $(4.25 \pm 1.29)$ was more than that in noncolonized children $(3.48 \pm 1.70)$. 
TABLE 2: Nursery's children data.

\begin{tabular}{|c|c|c|c|c|c|c|}
\hline \multirow[b]{2}{*}{ Variables } & \multirow[b]{2}{*}{ Groups } & \multicolumn{2}{|c|}{ H. influenzae } & \multirow[b]{2}{*}{ Unadjusted OR } & \multirow[b]{2}{*}{$95 \% \mathrm{CI}$} & \multirow[b]{2}{*}{$p$ value* } \\
\hline & & $\begin{array}{c}\text { Non } \mathrm{b} \text { or negative } \\
N(\%)\end{array}$ & $\begin{array}{l}\mathrm{Hib} \\
N(\%)\end{array}$ & & & \\
\hline \multirow{2}{*}{ Age group } & $1-3 \mathrm{yr}$ & $107(95.54)$ & $5(4.46)$ & 1 & \multirow{2}{*}{$0.23-2.20$} & \multirow{2}{*}{0.55} \\
\hline & $4-6 y r$ & $243(96.81)$ & $8(3.19)$ & 0.70 & & \\
\hline \multirow{2}{*}{ Gender } & Female & $170(94.97)$ & $9(5.03)$ & 1 & \multirow{2}{*}{$0.13-1.39$} & \multirow{2}{*}{0.15} \\
\hline & Male & $180(97.83)$ & $4(2.17)$ & 0.42 & & \\
\hline \multirow{2}{*}{ Family number } & $2-3$ & $225(96.57)$ & $8(3.43)$ & 1 & \multirow{2}{*}{$0.36-3.51$} & \multirow{2}{*}{0.84} \\
\hline & $\geq 4$ & $125(96.15)$ & $5(3.85)$ & 1.12 & & \\
\hline \multirow{2}{*}{ Father education } & Associate's degree, diploma, or less & $80(96.39)$ & $3(3.61)$ & 1.01 & \multirow{2}{*}{$0.27-3.77$} & \multirow{2}{*}{0.99} \\
\hline & BSc, MSc, or doctorate & $270(96.43)$ & $10(3.57)$ & 1 & & \\
\hline \multirow{2}{*}{ Mother education } & Associate's degree, diploma, or less & $69(94.52)$ & $4(5.48)$ & 1.81 & \multirow{2}{*}{$0.54-6.05$} & \multirow{2}{*}{0.34} \\
\hline & $\mathrm{BSc}, \mathrm{MSc}$, or doctorate & $281(96.90)$ & $9(3.10)$ & 1 & & \\
\hline \multirow{2}{*}{ Pantavalan intake } & No & $125(96.15)$ & $5(3.85)$ & 1.12 & \multirow{2}{*}{$0.36-3.51$} & \multirow{2}{*}{0.84} \\
\hline & Yes & $225(96.57)$ & $8(3.43)$ & 1 & & \\
\hline \multirow{2}{*}{ History } & Negative & $292(96.05)$ & $12(3.95)$ & 1 & \multirow{2}{*}{$0.05-3.29$} & \multirow{2}{*}{0.41} \\
\hline & Positive & $58(98.31)$ & $1(1.69)$ & 0.42 & & \\
\hline \multirow{2}{*}{ Smoking } & No & $337(96.29)$ & $13(3.71)$ & 1 & \multirow{2}{*}{$0.00-9.65$} & \multirow{2}{*}{1.00} \\
\hline & Yes & $13(100.00)$ & $0(0.00)$ & 1.46 & & \\
\hline \multirow{2}{*}{ Antibiotic } & No & $342(96.61)$ & $12(3.39)$ & 1 & \multirow{2}{*}{$0.41-30.80$} & \multirow{2}{*}{0.25} \\
\hline & Yes & $8(88.89)$ & $1(11.11)$ & 3.56 & & \\
\hline
\end{tabular}

*Simple and multiple logistic regression (forward method); $p<0.05$.

TABLE 3: Nonnursery's children data.

\begin{tabular}{|c|c|c|c|c|c|c|}
\hline \multirow[b]{2}{*}{ Variables } & \multirow[b]{2}{*}{ Groups } & \multicolumn{2}{|c|}{ H. influenzae } & \multirow[b]{2}{*}{ Unadjusted OR } & \multirow[b]{2}{*}{$95 \% \mathrm{CI}$} & \multirow[b]{2}{*}{$p$ value $^{*}$} \\
\hline & & $\begin{array}{c}\text { Non b or negative } \\
N(\%)\end{array}$ & $\begin{array}{l}\text { Hib } \\
N(\%)\end{array}$ & & & \\
\hline \multirow{2}{*}{ Age group } & $1-3 \mathrm{yr}$ & $338(99.12)$ & $3(0.88)$ & 1 & \multirow{2}{*}{$1.24-18.09$} & \multirow{2}{*}{0.02} \\
\hline & $4-6 \mathrm{yr}$ & $190(95.96)$ & $8(4.04)$ & 4.74 & & \\
\hline \multirow{2}{*}{ Gender } & Female & $269(98.53)$ & $4(1.47)$ & 1 & \multirow{3}{*}{$0.53-6.28$} & \multirow{3}{*}{0.35} \\
\hline & Male & $259(97.37)$ & $7(2.63)$ & 1.82 & & \\
\hline \multirow{3}{*}{ Family number } & $2-3$ & $246(99.19)$ & $2(0.81)$ & 1 & & \\
\hline & 4 & $234(97.50)$ & $6(2.50)$ & 3.15 & $0.63-15.78$ & 0.16 \\
\hline & $\geq 5$ & $48(94.12)$ & $3(5.88)$ & 7.69 & $1.25-47.24$ & 0.03 \\
\hline \multirow{2}{*}{ Father education } & Associate's degree, diploma, or less & $205(96.24)$ & $8(3.76)$ & 4.20 & \multirow{2}{*}{$1.10-16.02$} & \multirow{2}{*}{0.04} \\
\hline & $\mathrm{BSc}, \mathrm{MSc}$, or doctorate & $323(99.08)$ & $3(0.92)$ & 1 & & \\
\hline \multirow{2}{*}{ Mother education } & Associate's degree, diploma, or less & $203(96.21)$ & $8(3.79)$ & 4.27 & \multirow{2}{*}{$1.12-16.28$} & \multirow{2}{*}{0.03} \\
\hline & BSc, MSc, or doctorate & $325(99.09)$ & $3(0.91)$ & 1 & & \\
\hline \multirow{2}{*}{ Pantavalan intake } & No & $128(96.97)$ & $4(3.03)$ & 1.79 & \multirow{2}{*}{$0.51-6.20$} & \multirow{2}{*}{0.36} \\
\hline & Yes & $400(98.28)$ & $7(1.72)$ & 1 & & \\
\hline \multirow{2}{*}{ History } & Negative & $495(97.83)$ & $11(2.17)$ & 1 & \multirow{2}{*}{$0.00-6.27$} & \multirow{2}{*}{0.99} \\
\hline & Positive & $33(100.00)$ & $0(0.00)$ & $0.99^{*}$ & & \\
\hline \multirow{2}{*}{ Smoking } & No & $436(97.98)$ & $9(2.02)$ & 1 & \multirow{2}{*}{$0.22-4.95$} & \multirow{2}{*}{0.95} \\
\hline & Yes & $92(97.87)$ & $2(2.13)$ & 1.05 & & \\
\hline \multirow{2}{*}{ Antibiotic } & No & $519(97.92)$ & $11(2.08)$ & 1 & \multirow{2}{*}{$0.00-27.32$} & \multirow{2}{*}{1.00} \\
\hline & Yes & $9(100.00)$ & $0(0.00)$ & $3.89^{*}$ & & \\
\hline
\end{tabular}

*Simple and multiple logistic regression (forward method); $p<0.05$ 
In this study, none of the variables studied had a significant relationship with the colonization of $\mathrm{Hib}$, in nursery's children. In nonnursery's children with $H i b$, age 4 to 6 years $(p=0.02)$, family number $(p=0.03)$, lower educational level of father $(p=0.04)$, and mother's level of education $(p=0.03)$ correlated with increased odds of colonization.

Although there is controversy about the correlation between the $\mathrm{Hib}$ carriage rate and other factors, such as gender, number of siblings $<5$ years old, and recent respiratory disease [23]. In Puig et al.'s study, the association between gender and respiratory problems with $H$. influenzae colonization is not shown. [23]. With regard to CDC, exposure factors include household crowding, large household size, child care attendance, low socioeconomic status, low parental education levels, and school-aged sibling effect on Hib [7]. Similar to the CDC results, this study showed that in nonnursery's children, the rate of Hib colonization was related to the family number and lower educational level of father and mother. Also, in one study, in China in 2016, inverse association between household size and Hib carriage was shown. So Hib carriage was more in children with more siblings compared to single children. It indicated possible effects from human crowding. It seems crowding and having more children in the family are reasons for more Hib carriage in developing countries than in developed countries [26].

According to a study by Jalali et al. in 2014, the rate of $H$. influenzae carriers is high (28\%) in children under 6 years of age in Iran and similar to unvaccinated countries. In this study, 533 mucus samples were obtained by nasopharyngeal swabs from children under 6 years old in 4 nursery centers in Tehran or refereed to the Children's Medical Center of Tehran. The $H$. influenzae diagnosis was performed by standard biochemical tests and confirmed by PCR assay. That result showed the rate of $H$. influenzae carriage was linked to age and respiratory infection diseases. The carriage rate was highest in children aged 25-48 months and decreased with increasing age [27].

This study indicated that $H i b$ vaccine, as a constituent of pentavalent vaccine, successfully reduced $\mathrm{Hib}$ nasopharyngeal colonization.

One of the limitations of this study was the difficulty of swab sampling from children under two years. This can affect the outcome of the study. Moreover, in this study, the use of antibiotics was not investigated. This variable may affect the rate of Hib cloning.

\section{Conclusion}

Vaccination of Hib conjugate has been displayed to reduce the pharyngeal carriage rate of $\mathrm{Hib}$ [22]. Our results showed that compared to the carrier group, which was carried out before vaccinations in the same age group in Tehran, Iran, about 3 times (2.6 compared to 7.5), the carrier rate decreased. Thus, the vaccination has been able to reduce about $60 \%$ of the pharyngeal carriers. In unvaccinated children representing a $3.6 \%$ pharyngeal carrier, it depicts the topic of herd immunity. Following vaccinations in the target group, the carrier rate has also declined in the groups that did not receive the vaccine.
According to the $2.6 \%$ rate of $H$. influenzae after vaccination program and comparison with the rate below $1 \%$ in other countries, it was recommended that future studies investigate colonization in Iranian children.

\section{Data Availability}

The required data are given in the text of the article.

\section{Conflicts of Interest}

There are no conflicts of interest.

\section{Acknowledgments}

This project was funded by the World Health Organization (WHO) and Shahid Beheshti University of Medical Sciences, Tehran, Iran (Gran no. 247).

\section{References}

[1] A. Fahimzad, A. Karimi, A. Alborzi, S. R. Tabatabae, F. Jadali, and M. Sharifian, "Antibiotic susceptibility patterns in h. Influenzae type b isolated from healthy children oropharynx in day care centers of tehran," Iranian Journal of Pediatrics, vol. 17, Supplement 2, 2007.

[2] F. Shirvani, R. Arjmand, M. Golami et al., "Oropharyngeal colonization of Haemophilus influenzae type b and serologic response after administration of third dose of pentavalent vaccine to 12-month-old children in Karaj, Iran, 2016," Archives of Pediatric Infectious Diseases, vol. 7, no. 1, article e82238, 2019.

[3] D. F. Butler and A. L. Myers, "Changing epidemiology of Haemophilus influenzae in children," Infectious Disease Clinics of North America, vol. 32, no. 1, pp. 119-128, 2018.

[4] J. R. Gilsdorf, "What the pediatrician should know about nontypeable Haemophilus influenzae," The Journal of Infection, vol. 71, Suppl 1, pp. S10-S14, 2015.

[5] M. Romaneli, A. T. Tresoldi, R. M. Pereira, M. T. Garcia, C. C. Hofling, and M. R. Resende, "Invasive non-type b Haemophilus influenzae disease: report of eight cases," Revista Paulista de Pediatria, vol. 37, no. 2, pp. 156-160, 2019.

[6] E. Williams, J. Lewis, T. John et al., "Haemophilus influenzae type b carriage and novel bacterial population structure among children in urban Kathmandu, Nepal," Journal of Clinical Microbiology, vol. 49, no. 4, pp. 1323-1330, 2011.

[7] Centers for Disease Control and Prevention, "Haemophilus influenzae type b," in Epidemiology and Prevention of Vaccine-Preventable Diseases, J. Hamborsky, K. Andrew, and C. S. Wolfe, Eds., pp. 119-134, Public Health Foundation, Washington D.C., 13th edition, 2015.

[8] B. Wahl, K. L. O'Brien, A. Greenbaum et al., "Burden of Streptococcus pneumoniae and Haemophilus influenzae type b disease in children in the era of conjugate vaccines: global, regional, and national estimates for $2000-15$," The Lancet Global Health, vol. 6, no. 7, pp. e744-e757, 2018.

[9] R. C. Zanella, M. C. Brandileone, A. L. Andrade et al., "Evaluation of Haemophilus influenzaetype b carrier status among children 10 years after the introduction of Hib vaccine in Brazil," Memorias do Instituto Oswaldo Cruz, vol. 110, no. 6, pp. 755-759, 2015. 
[10] L. L. Hammitt, R. J. Crane, A. Karani et al., "Effect of Haemophilus influenzae type b vaccination without a booster dose on invasive $H$ influenzae type $b$ disease, nasopharyngeal carriage, and population immunity in Kilifi, Kenya: a 15-year regional surveillance study," The Lancet Global Health, vol. 4, no. 3, pp. e185-e194, 2016.

[11] S. Wang, M. Tafalla, L. Hanssens, and J. Dolhain, "A review of Haemophilus influenzae disease in Europe from 2000-2014: challenges, successes and the contribution of hexavalent combination vaccines," Expert Review of Vaccines, vol. 16, no. 11, pp. 1095-1105, 2017.

[12] M. Giufrè, M. Fabiani, R. Cardines et al., "Increasing trend in invasive non-typeable Haemophilus influenzae disease and molecular characterization of the isolates, Italy, 2012 -2016," Vaccine, vol. 36, no. 45, pp. 6615-6622, 2018.

[13] S. Collins, D. Litt, R. Almond et al., "Haemophilus influenzae type b (Hib) seroprevalence and current epidemiology in England and Wales," The Journal of Infection, vol. 76, no. 4, pp. 335-341, 2018.

[14] Centers for Disease Control and Prevention, "Active bacterial core surveillance report, Emerging Infections Program Network, Haemophilus influenza 2015," 2015, https://www.cdc .gov/abcs/reports-findings/survreports/hib15.html.

[15] S. Suga, N. Ishiwada, Y. Sasaki et al., "A nationwide population-based surveillance of invasive Haemophilus influenzae diseases in children after the introduction of the Haemophilus influenzae type b vaccine in Japan," Vaccine, vol. 36, no. 38, pp. 5678-5684, 2018.

[16] B. Wahl, A. Sharan, M. Deloria Knoll et al., "National, regional, and state-level burden of Streptococcus pneumoniae and Haemophilus influenzae type b disease in children in India: modelled estimates for 2000 -15," The Lancet Global Health, vol. 7, no. 6, pp. e735-e747, 2019.

[17] M. Karami, P. Ameri, J. Bathaei et al., "Adverse events following immunization with pentavalent vaccine: experiences of newly introduced vaccine in Iran," BMC Immunology, vol. 18, no. 1, p. 42, 2017.

[18] T. Ueyama, Y. Kurono, K. Shirabe, M. Takeshita, and G. Mogi, "High incidence of Haemophilus influenzae in nasopharyngeal secretions and middle ear effusions as detected by PCR," Journal of Clinical Microbiology, vol. 33, no. 7, pp. 1835-1838, 1995.

[19] V. Eton, A. Schroeter, L. Kelly, M. Kirlew, R. S. W. Tsang, and M. Ulanova, "Epidemiology of invasive pneumococcal and Haemophilus influenzae diseases in Northwestern Ontario, Canada, 2010 -2015," International Journal of Infectious Diseases, vol. 65, pp. 27-33, 2017.

[20] A. Saito, H. Sumita, Y. Minohara et al., "Nasopharyngeal Hib carriage among healthy children attending daycare centers in Yokohama after one year of a publicly funded vaccine program," Kansenshōgaku Zasshi, vol. 89, no. 1, pp. 30-36, 2015.

[21] R. C. Zanella, M. C. C. Brandileone, A. L. Andrade et al., "Evaluation of Haemophilus influenzae type b carrier status among children 10 years after the introduction of Hib vaccine in Brazil," Memórias do Instituto Oswaldo Cruz, vol. 110, no. 6, pp. 755-759, 2015.

[22] M. Giufrè, L. Daprai, R. Cardines et al., "Carriage of Haemophilus influenzae in the oropharynx of young children and molecular epidemiology of the isolates after fifteen years of $H$. influenzae type b vaccination in Italy," Vaccine, vol. 33, no. 46, pp. 6227-6234, 2015.
[23] C. Puig, S. Marti, A. Fleites et al., "Oropharyngeal colonization by nontypeable Haemophilus influenzae among healthy children attending day care centers," Microbial Drug Resistance, vol. 20, no. 5, pp. 450-455, 2014.

[24] A. Odutola, M. Antonio, O. Owolabi et al., "Comparison of the prevalence of common bacterial pathogens in the oropharynx and nasopharynx of Gambian infants," PLoS One, vol. 8, no. 9, article e75558, 2013.

[25] A. Karimi, A. Alborzi, A. Fahimzad et al., "Prevalence of oropharyngeal colonization by Haemophilus influenzae type b in Iranian children," Eastern Mediterranean Health Journal, vol. 15, no. 3, pp. 544-548, 2009.

[26] J. Hu, X. Sun, Z. Huang et al., "Streptococcus pneumoniae and Haemophilus influenzae type b carriage in Chinese children aged 12-18 months in Shanghai, China: a cross-sectional study," BMC Infectious Diseases, vol. 16, no. 1, p. 149, 2016.

[27] P. Jalali, S. F. Mousavi, and N. Rezaei, "Carriage rate of nasopharyngeal Haemophilus influenzae among children under 6 years old in Tehran, Iran," Journal of Medical Microbiology And Infectious Diseases, vol. 2, pp. 23-27, 2014. 